\title{
Serum lipoprotein binding by Treponema pallidum: possible role for proteoglycans
}

\author{
J F ALDERETE, J B BASEMAN
}

From the Department of Microbiology, University of Texas Health Science Center, San Antonio, Texas, USA

SUMMARY Acquisition by the syphilis spirochaete, Treponema pallidum, of radioiodinated total human plasma lipoprotein and lipoprotein subfractions was examined. Time dependent and saturation binding kinetics were observed for total lipoproteins and subfractions, including high density lipoproteins, low density lipoproteins (LDL), and very low density lipoproteins. All subfractions competed equally well in binding iodinated total lipoproteins and individual subfractions, but apoproteins common to all subfractions were ineffective in inhibiting lipoprotein acquisition. The interaction of LDL with $T$ pallidum was studied further and, interestingly, the presence of $17 \%$ sulphated dextran sulphate (DS) in the reaction mixture containing treponemes and LDL resulted in up to 172 times more LDL being bound by live treponemes. Biological variability was observed in the extent of increased LDL bound in the presence of $17 \%$ sulphated DS by preparations of $T$ pallidum isolated from different infected rabbits. Saturation kinetics of iodinated LDL acquisition was obtained in the presence of $17 \%$ sulphated DS but not $1 \%$ sulphated DS. Other proteoglycan molecules, such as chondroitin sulphate, hyaluronic acid and heparin, and fibronectin, the extracellular matrix protein targeted by treponemes in parasitism of host cells and tissues neither diminished nor enhanced LDL binding by live treponemes. Only $5 \%$ and $10 \%$ of associated radioactivity was released from treponemal surfaces after $T$ pallidum was incubated with iodinated LDL and $17 \%$ sulphated-DS for 15 and 30 minutes, respectively. These data show binding and possible internalisation of host lipoproteins by $T$ pallidum, which may be mediated by sulphated proteoglycan. Sulphated proteoglycans accumulate during $T$ pallidum infections of host cells.

Treponema pallidum is the causative agent of syphilis, which is characterised by a complex relation between host and parasite. The ability of treponemes to acquire host derived plasma molecules selectively and avidly has been of particular interest to us. ${ }^{12}$ In addition to possibly protecting the treponemes from specific host immune surveillance, a coat of host derived macromolecules may also play a part in neutralising nonspecific defence mechanisms, such as host proteases, and provide nutrients essential for treponemal survival. ${ }^{34}$ Earlier studies showed that up to 12 human plasma proteins were bound by $T$ pallidum, but not by the cultivable avirulent spirochaete, $T$ phagedenis biotype Reiter. ${ }^{2}$ A stepwise approach was developed to

Address for reprints: Dr John F Alderete, Department of Microbiology, University of Texas Health Science Center at San Antonio, 7703 Floyd Curl Drive, San Antonio, Texas 78284-7758, USA

Accepted for publication 27 January 1989 identify these host macromolecules, which led to the discovery of fibronectin as a possible receptor that mediated treponemal binding to host cells and tissues. ${ }^{25}$ More recently, the specific acquisition of iron via lactoferrin and transferrin associations was investigated using live $T$ pallidum organisms. ${ }^{3}$ These data suggest that the binding of host proteins by $T$ pallidum is relevant to the establishment and progression of syphilis.

Because plasma components appear to enhance the in vitro survival of these microbial pathogens, ${ }^{4}$ we examined the interactions between $T$ pallidum and lipoproteins, another class of plasma components that has received little or no attention in connection with this parasite. We here provide evidence of human plasma lipoprotein binding and possible internalisation. Furthermore, data indicate a role for sulphated proteoglycan molecules. Glycosaminoglycans are known to accumulate and coat treponemes shortly after and during infection of host cells in vitro and 
rabbit testes in vivo. ${ }^{6-8}$ These observations are important in view of the possible relevance of mucopolysaccharides (derived from the parasite or the host) to the growth and multiplication of treponemes in vitro and in vivo.

\section{Materials and methods}

\section{BACTERIA}

Procedures for harvesting treponemes from the infected testes of New Zealand white rabbits have been described previously.' Organisms were clarified from host debris, and the bacteria were pelleted and resuspended at a density of $1.2 \times 10^{9}$ organisms in $500 \mu \mathrm{l}$ phosphate buffered saline (PBS) in siliconised $1.5 \mathrm{ml}$ microfuge tubes. Only freshly extracted treponemes were used for all experiments.

\section{TOTAL PLASMA LIPOPROTEINS AND LIPOPROTEIN} SUBFRACTIONS

Lipoproteins were prepared from pooled normal human plasma in $0.1 \%$ (weight/volume) ethylenediaminetetraacetic acid (EDTA). Very low density lipoproteins were first removed by ultracentrifugation, in a Beckman $60 \mathrm{Ti}$ rotor $\left(\right.$ at $175000 \times \mathrm{g}$ ) at $10-15^{\circ} \mathrm{C}$ for 24 hours. ${ }^{910}$ After removal of the very low density lipoproteins, the remaining plasma was adjusted with solid potassium bromide to a density of $1.063 \mathrm{~g} / \mathrm{ml}$ and centrifuged for an additional 24 hours. The low density lipoprotein (LDL) was removed, and the solution adjusted with potassium bromide to 1.215 $\mathrm{g} / \mathrm{ml}$. After being centrifuged for 24 hours, the high density lipoprotein fraction was recovered. All lipoprotein subfractions were then extensively dialysed against several changes (4 l each) of PBS (137 $\mathrm{mmol} / 1$ sodium chloride, $2.7 \mathrm{mmol} / 1$ potassium chloride, $4.6 \mathrm{mmol} / \mathrm{l}$ dibasic sodium phosphate, and $1.5 \mathrm{mmol} / 1 \mathrm{monobasic}$ potassium phosphate), $\mathrm{pH}$ $7 \cdot 4 .^{1 ~}{ }^{\text {II }}$ Lipoproteins were used immediately or stored at $4^{\circ} \mathrm{C}$ no longer than three days after isolation.

\section{RADIOIODINATION OF LIPOPROTEINS}

All lipoprotein subfractions and LDL were radioiodinated using an oxidative iodine monochloride technique described previously. ${ }^{12}$ Lipoprotein solutions $(10 \mathrm{mg} / \mathrm{ml})$ were mixed with an equal volume of $2 \mathrm{~mol} / \mathrm{l}$ glycine-sodium hydroxide buffer, $\mathrm{pH} \mathrm{10}$, and $1.0 \mathrm{mCi}$ sodium radioiodide $\left(\mathrm{Na}^{125} \mathrm{I}\right.$; Amersham Corporation, Arlington Heights, Illinois, USA) was then added. To the mixture was added $300 \mu \mathrm{l} 100$ $\mathrm{mmol} / 1$ sodium iodide and $10 \mathrm{mmol} / 1$ sodium iodate in $7 \mathrm{~mol} / \mathrm{l}$ hydrochloric acid diluted $1: 100$ in $2 \mathrm{~mol} / 1$ sodium chloride. After one minute the mixture was placed on a Sephadex G-25 column $(1 \times 10 \mathrm{~cm})$ equilibrated in PBS containing $0.01 \%$ EDTA. The efficiency of iodination was assessed by trichloroacetic acid precipitation," 12 and lipid associated radioactivity was measured by chloroform/methanol $(2: 1$, $\mathrm{vol} / \mathrm{vol}$ ) extraction. ${ }^{10}$ This was necessary to show that treponemal associations with lipoproteins were not solely the result of binding apoproteins of lipoproteins.

\section{LIPOPROTEIN BINDING ASSAYS}

Motile treponemes that had been harvested freshly from infected rabbit testes were pelleted and resuspended to about $2.4 \times 10^{9}$ organisms $/ \mathrm{ml}$. Different amounts of radioiodinated total lipoproteins or lipoprotein subfractions were added in $0.5 \mathrm{ml}$ aliquots to siliconised microfuge tubes containing $1.2 \times 10^{9} \mathrm{~T}$ pallidum in PBS." The final volume was adjusted to $1.0 \mathrm{ml}$ with PBS. After being incubated for different times and temperatures, treponemes were pelleted at $17500 \times g$ and washed twice in ice cold PBS, and the pelleted organisms were resuspended and placed in another microfuge tube for avidly associated radioactivity to be measured.

Competition experiments were performed identically except that unlabelled proteins were added to the treponemes immediately before the addition of iodinated lipoproteins."

For experiments assessing the incorporation of iodinated lipoproteins, treponemes were processed as above followed by treatment for 30 minutes with 250 $\mu \mathrm{g}$ trypsin. These conditions are known to release treponemal surface proteins..$^{13}$ Soybean trypsin inhibitor was added immediately to organisms trypsinised for different times, which were then washed, and associated radioactivity was counted.

\section{Results}

BINDING OF TOTAL LIPOPROTEINS AND

\section{LIPOPROTEIN SUBFRACTIONS}

Initial experiments were conducted using iodinated total lipoproteins and the three lipoprotein subfractions. After being incubated for 5,10 , or 30 minutes with $T$ pallidum, $100 \mu \mathrm{g}$ of total lipoproteins and all lipoprotein subfractions showed similar levels of binding to $1.2 \times 10^{9}$ live motile organisms. Low density lipoprotein bound two to three times more than the other lipoprotein subfractions. Individual lipoprotein subfractions showed no differences in levels of treponemal acquisition at $4^{\circ} \mathrm{C}, 22^{\circ} \mathrm{C}$, or $37^{\circ} \mathrm{C}$ or at a range of 6.0 to $7.5 \mathrm{pH}$. Saturation binding occurred within 15 minutes at each temperature. Competition experiments were also performed, and all subfractions gave diminished binding of iodinated lipoproteins at 4:1 ratios of unlabelled lipoprotein subfractions and $100 \mu \mathrm{g}$ iodinated total lipoproteins. These data suggested that treponemes interacted with human plasma lipoproteins in a specific fashion, based 


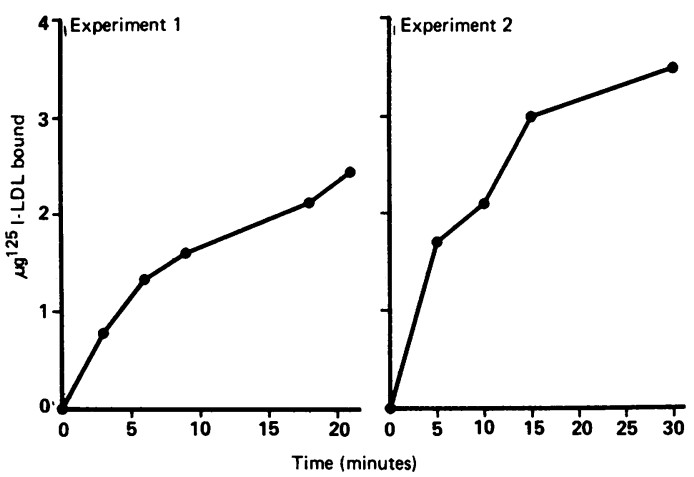

Fig 1 Kinetics of low density lipoprotein ( $L D L)$ binding to $T$ pallidum using $1.2 \times 10^{9}$ organisms incubated with $100 \mu \mathrm{g}$ iodinated LDL in a final volume of $1 \mathrm{ml}$ in phosphate buffered saline (PBS). Each point represents a different reaction, and each experiment uses organisms from a different rabbit.

on time dependent binding and saturation kinetics. Furthermore, a component on treponemal surfaces may possibly be recognised by all lipoprotein subfractions.

\section{LDL ASSOCIATIONS WITH TREPONEMES}

LDL acquisition by $T$ pallidum was characterised in more detail to understand better the nature and specificity of the interactions with this class of plasma molecule. Figure 1 shows the time course of binding iodinated LDL in two representative experiments. Similar kinetics of binding were also seen at $4^{\circ} \mathrm{C}$ and $22^{\circ} \mathrm{C}$ (data not shown). After incubating treponemes with iodinated LDL for 15 minutes, trypsin treatment removed only $5 \%$ of cell associated radioactivity (data not shown), which suggested the possible internalisation of lipoproteins by treponemes.

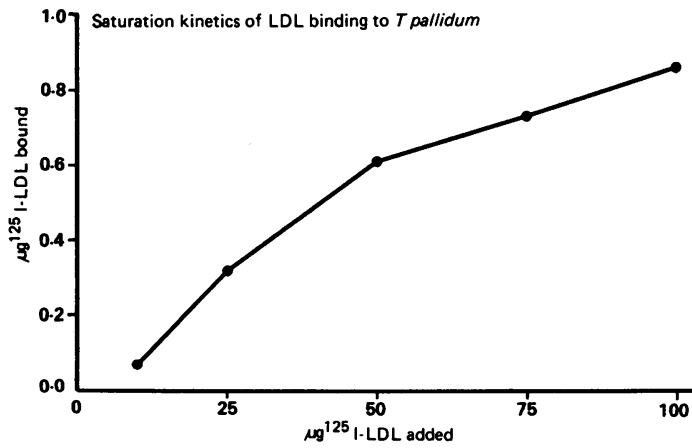

Fig 2 Acquisition of iodinated low density lipoproteins ( $L D L)$ by treponemes depending on concentration of $L D L$. Treponemes were incubated at $4^{\circ} \mathrm{C}$ for 15 minutes with increasing concentrations of $L D L$.
Figure 2 shows the increased binding of iodinated LDL when additional amounts were added to $1.2 \times$ $10^{9}$ treponemes. This experiment was performed at $4^{\circ} \mathrm{C}$ to minimise the possible internalisation of LDL. A gradual levelling off was always seen after $50 \mu \mathrm{g}$ iodinated LDL had been added.

We were unsuccessful in attempts to show the inhibition of iodinated LDL binding by live treponemes with several apoproteins found in lipoprotein subfractions. ${ }^{9112}$ The incubation of treponemes with up to $200 \mu \mathrm{g}$ purified apoproteins A, B, CIII, or E for 5,15 , or 30 minutes before adding iodinated LDL showed levels of LDL bound that were similar to those bound to control treponemes incubated without apoproteins. These data indicated that the mode of treponemal binding of LDL and the other lipoprotein subfractions may not have been mediated by specific apoproteins of lipoprotein, as has been reported for Trichomonas vaginalis, another sexually transmitted microorganism. ${ }^{11}$

\section{HIGHLY SULPHATED DEXTRAN SULPHATE}

INCREASES DRAMATICALLY AND SPECIFICALLY

THE AMOUNT OF LDL BOUND BY $T$ pallidum

Treponemal parasitism of host cells and rabbit testes results in the accumulation of glycosaminoglycans, especially chondroitin sulphate and hyaluronic acid, on $T$ pallidum surfaces. ${ }^{6-8}$ Because some proteoglycans are known to bind lipoproteins in a specific way, ${ }^{14}$ and because they readily coat treponemes, ${ }^{6-8}$ we reasoned

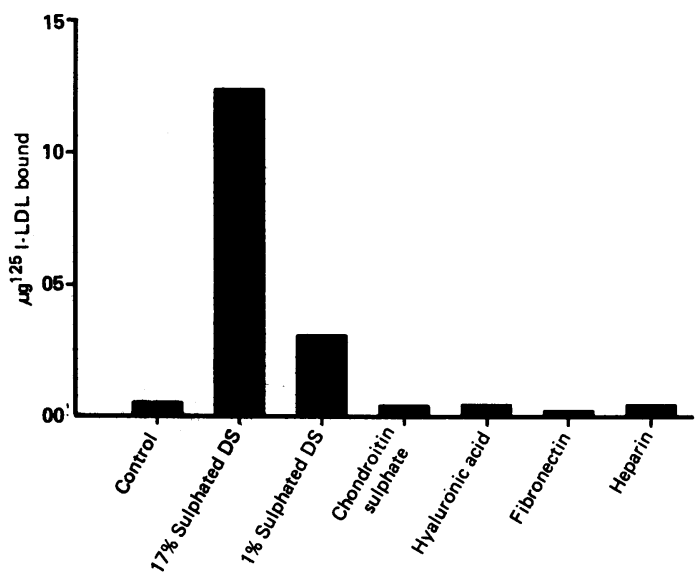

Fig 3 Representative experiment showing levels of iodinated low density lipoproteins ( $L D L)$ bound by treponemes in the absence (control) or presence of $100 \mu \mathrm{g}$ exogenous dextran sulphate (DS), proteoglycan molecules (chondroitin sulphate and heparin), or human plasma fibronectin. Concentrations of proteoglycan and fibronectin ranging from $10 \mu \mathrm{g}$ to $500 \mu \mathrm{g}$ (Sigma, St Louis, Missouri, USA) were used, and no effect on LDL binding was detected at any concentration. 


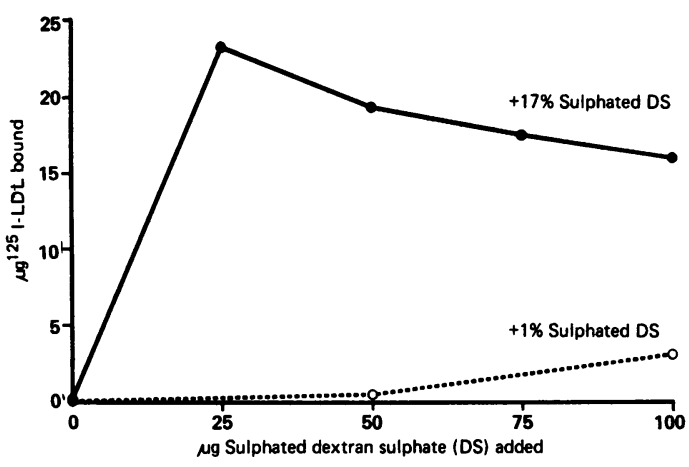

Fig 4 Comparison of iodinated low density lipoproteins (LDL) binding using different concentrations of $17 \%$ sulphated dextran sulphate (DS) and 1\% sulphated DS. For this experiment DS was added to treponemes just before incubation with iodinated LDL for 15 minutes.

that they might also influence the extent of lipoprotein association with $T$ pallidum.

Figure 3 shows a representative experiment during which treponemes were pretreated with $100 \mu \mathrm{g}$ of various substances before $100 \mu \mathrm{g}$ iodinated $\mathrm{LDL}$ was added. To our surprise only $17 \%$ sulphated dextran sulphate (DS), and to a lesser extent $1 \%$ sulphated DS, resulted in higher levels of iodinated LDL being bound than by controls without exogenous additions or with other proteoglycans, such as chondroitin sulphate, hyaluronic acid, or heparin. Fibronectin, the plasma glycoprotein mediating treponemal attachment to cells and extracellular matrix, ${ }^{25}$ also failed to affect the

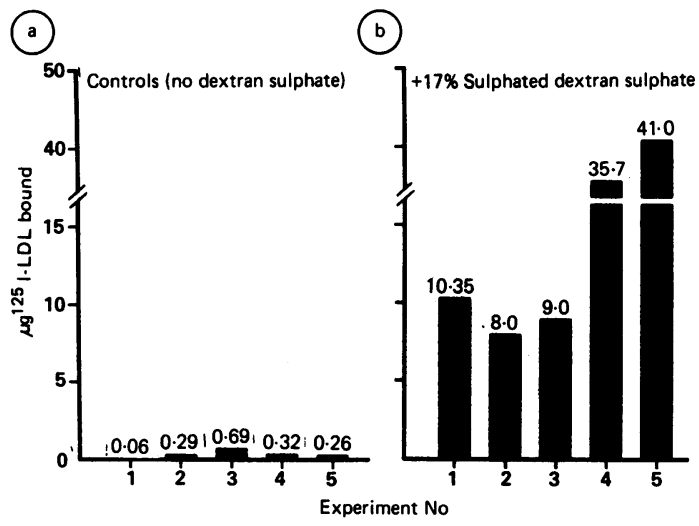

Fig 5 Experiments showing variations in levels of iodinated low density lipoproteins ( $L D L$ ) bound to treponemes obtained from testes of five different rabbits in the absence (a) and presence (b) of $17 \%$ sulphated dextran sulphate (DS). For these experiments $25 \mu \mathrm{g}$ of $17 \%$ sulphated DS was added to the reaction mixture. Experiment 1 shows 172-fold greater binding of iodinated LDL than by the control that contained organisms from the same rabbit (a).

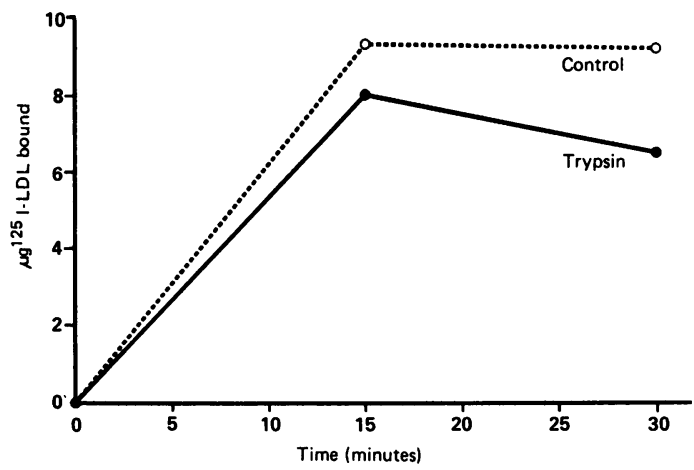

Fig 6 Time course binding of iodinated low density lipoprotein ( $L D L)$ to $T$ pallidum organisms in the presence of $25 \mu \mathrm{g} 17 \%$ sulphated dextran sulphate. The level of $L D L$ bound by treponemes was consistent with levels observed in other experiments. Treponemes of duplicate samples handled identically were also pelleted, as described in Materials and methods, and treated with $250 \mu \mathrm{g}$ trypsin freshly prepared in phosphate buffered saline for 30 minutes. These protease treatment conditions are known to cause the release of treponemal surface proteins.

levels of LDL bound. Finally, the addition of 100 $\mathrm{mmol} / \mathrm{l}$ each of magnesium sulphate and ammonium sulphate did not alter the levels of LDL acquisition in the presence or absence of enhanced LDL binding mediated by $17 \%$ sulphated DS.

We than added different amounts of $17 \%$ sulphated DS to treponemes, and then added $100 \mu \mathrm{g}$ iodinated LDL. Figure 4 shows that maximum levels of LDL binding were achieved with $25 \mu \mathrm{g}$ of this highly sulphated molecule. Dramatically greater binding of LDL was seen with all concentrations of $17 \%$ sulphated DS than with $1 \%$ sulphated DS, which indicated that the highly sulphated nature of DS was important in increasing associations between lipoproteins and treponemes.

The effect of $17 \%$ sulphated DS in enhancing LDL acquisition was further confirmed by five additional separate experiments (fig 5) using organisms derived from the testes of different rabbits. The presence of 25 $\mu \mathrm{g}$ highly sulphated DS gave LDL levels up to 170 times higher than those observed for the control samples (fig 4). These data also show the biological variability between different treponemal populations.

Figure 6 presents data on the time course binding of LDL in the presence of $25 \mu \mathrm{g} 17 \%$ sulphated DS. These results are consistent with the earlier observation showing maximum levels of lipoproteins bound to treponemes for 10 to 15 minutes (fig 1). Trypsinisation of live treponemes after 15 and 30 minutes incubation with iodinated lipoproteins and $17 \%$ sulphated DS also failed to remove $80 \%$ to $85 \%$ of the radioactivity, which suggested the internalisation of the LDL bound to the surface. 


\section{Discussion}

The biology of $T$ pallidum lipid metabolism remains poorly understood. Except for studies defining the effects of fatty acids on the retention of motility and viability of treponemes ${ }^{15-17}$ or analysing the fatty acid composition of treponemal membranes, ${ }^{18}$ very little data are available regarding mechanisms by which these micro-organisms synthesise ${ }^{19}$ or sequester these important nutrients from the host environment.

Our goal in the past several years has been to examine the specificity and importance of acquiring host protein by $T$ pallidum organisms. ${ }^{12}$ We believe that beneficial properties are conferred on treponemes by, for example, the binding of specific human plasma proteins. We have characterised the possible role of fibronectin bound to surfaces of treponemes in mediating cytadherence, ${ }^{25}$ and those findings allowed us to confirm that three treponemal surface proteins were adhesins important for fibronectin binding. ${ }^{2}$ More recent observations documented the iron uptake by $T$ pallidum after receptor mediated lactoferrin and transferrin binding. ${ }^{3}$ The previous studies and the report published here may prove to be important not only for studying events that lead to the initial establishment of infection but also in explaining the difficulty of cultivating the organisms in vitro. ${ }^{1720}$

Initially it appeared that lipoprotein associations with treponemes were ron-specific, as individual lipoprotein subfractions were ineffective in inhibiting the binding of total lipoproteins. Apoproteins at high concentrations also failed to inhibit any of the lipoprotein subfractions, which indicated an absence of receptors on $T$ pallidum for the specific recognition of lipoprotein components. Evaluating various proteoglycan substrates during syphilis infection, however, including glycosaminoglycans such as hyaluronic acid and chondroitin sulphate, ${ }^{6-821}$ led to the observation that $17 \%$ sulphated DS enhances specific lipoprotein acquisition in a specific fashion. The requirement for the high degree of sulphation and specificity for $17 \%$ sulphated DS were important, as $1 \%$ sulphated DS and all proteoglycan molecules tested showed no enhancement of lipoprotein binding (figs 3 and 5). Increasing amounts of unlabelled lipoproteins added to iodinated LDL before incubation with organisms suspended in $17 \%$ sulphated DS also gave stoichiometric inhibition of iodinated LDL bound by treponemes (data not shown).

The presence of large amounts of these proteoglycan molecules after syphilis infection has received extensive attention in published reports, ${ }^{6-821}$ although the role of these substances in the establishment and pathogenesis of syphilis remains unknown. The treponemes are known to coat themselves with glycosaminoglycans during syphilis infection. ${ }^{8}$
Overall, except for identifying chondroitin sulphate and hyaluronic acid as predominant species of proteoglycans detected during infection, ${ }^{6-8}$ little chemical characterisation has been performed to define accurately the true composition of all possible proteoglycans detected on parasitised tissue cells ${ }^{7}$ or in sites of infection. ${ }^{21}$ The degree to which $17 \%$ sulphated DS permits increased lipoprotein binding, up to 170 times that seen in the absence of this molecule (fig 4), strongly indicates that a particular class of proteoglycans may serve similar functions in vivo. These data strengthen the argument that coating $T$ pallidum with glycosaminoglycans ${ }^{6-821}$ and other important host proteins ${ }^{12}$ is indeed an important feature that can confer numerous properties on the treponeme during the establishment and pathogenesis of syphilis. Data suggest that a layer of proteoglycans may act as an attractant or matrix for binding specific lipoproteins and possibly other nutrients.

Cholesterol, a component of all lipoprotein subfractions, has been found to be a main constituent of the lipids of $T$ pallidum..$^{18}$ Although some fatty acids can be synthesised by treponemes ${ }^{19}$ no evidence exists for de novo synthesis of this sterol. Finally, consistent with our data is the known incorporation of plasma cholesterol by $T$ phagedenis, ${ }^{22}$ which shows that plasma cholesterol may indeed represent a source for this nutrient in treponemes.

The study published here also shows the biological variability that exists between treponemes obtained from different animals (fig 4), which is consistent with our earlier reports studying the binding of host molecules by $T$ pallidum..$^{13}$ These variations indicate that treponemal populations are heterogeneous regarding their surface properties, and that passage through individual rabbits influences the particular subpopulation of treponemes that survive.

This work was supported by Public Health Service grant AI-19566 and by Research Career Development Award K04 AI-00584 from the National Institutes of Allergy and Infectious Diseases to JFA. We thank Ms Diana Hinojosa for her secretarial help and Larry Benavides for his technical help.

\section{References}

1 Alderete JF, Baseman JB. Surface-associated host proteins on virulent Treponema pallidum. Infect Immun 1979;26:1048-56.

2 Peterson KM, Baseman JB, Alderete JF. Treponema pallidum receptor binding proteins interact with fibronectin. J Exp Med 1983;157:1958-70.

3 Alderete JF, Peterson KM, Baseman JB. Affinities of Treponema pallidum for human lactoferrin and transferrin. Genitourin Med 1988;64:359-63.

4 Nelson RA. Factors affecting the survival of Treponema pallidum in vitro. Am J Hyg 1948;48:120-32.

5 Thomas DD, Baseman JB, Alderete JF. Fibronectin mediates Treponema pallidum cytadherence through recognition of fibronectin cell-binding domain. J Exp Med 1985;161:514-25. 
6 Fitzgerald TJ, Johnson RC, Ritzi DM. Relationship of Treponema pallidum to acidic mucopolysaccharides. Infect Immun 1979; 24:252-60.

7 Fitzgerald TJ, Johnson RC. Mucopolysaccharides of Treponema pallidum. Infect Immun 1979;24:261-68.

8 Fitzgerald TJ, Miller JN, Repesh LA, Rice M, Urquhart A. Binding of glycosaminoglycans to the surface of Treponema pallidum and subsequent effects on complement interactions between antigen and antibody. Genitourin Med 1985;61:13-20.

9 Havel RJ, Eder HA, Bragdon JP. The distribution and chemical composition of ultracentrifugally separated lipoproteins in human serum. J Clin Invest 1955;34:1345-53.

10 Folch J, Lees M, Sloane GH. A simple method for the isolation and purification of total lipids from animal tissues. J Biol Chem 1957;226:497-509.

11 Peterson KM, Alderete JF. Trichomonas vaginalis is dependent on uptake and degradation of human low density lipoproteins. JExp Med 1984;160:1261-72.

12 McFarlane AS. Metabolism of plasma proteins. In: Munra HN, Allison JB, eds. Mammalian protein metabolism. New York: Academic Press, 1982:297-341.

13 Alderete JF, Baseman JB. Surface characterization of virulent Treponema pallidum. Infect Immun 1980;30:814-23.

14 Fransson L-A, Johansson BG. Glycosaminoglycan-lipoprotein interactions: 1. Effect of uronic acid composition and charge density of the glycan. International Journal of the Biology of Macromolecules 1981;3:25-30.

15 Matthews HM, Jenkin HM, Crilly K, Sandok PL. Effects of fatty acids on motility retention by Treponema pallidum in vitro. Infect Immun 1978;19:814-21.

16 Nevin TA, Guest WJ, Geller RC. Response of Treponema pallidum to certain nutriles. British Journal of Venereal Diseases 1968;44:274-6.

17 Kimm GE, Allen RH, Morton HJ, Morgon JF. Studies on the in vitro survival of virulent Treponema pallidum. Am J Hyg 1962; 75:339-46.

18 Matthews HM, Yang T-K, Jenkins HM. Unique lipid composition of Treponema pallidum (Nichols virulent strain). Infect Immun 1979;24:713-9.

19 Schiller NL, Cox CD. Catabolism of glucose and fatty acids by virulent Treponema pallidum. Infect Immun 1977;16:60-8.

20 Fieldsteel AH, Becker FA, Stout JG. Prolonged survival of virulent Treponema pallidum (Nichols strain) in cell-free and tissue culture systems. Infect Immun 1977;18:173-82.

21 van der Sluis JJ, van Dijk G, Boer M, Stolz E, van Joost T. Mucopolysaccharides in suspensions of Treponema pallidum extracted from infected rabbit testes. Genitourin Med 1985;61: $7-12$. 